Cabero-Almenara, J., Barroso-Osuna, J., Palacios Rodríguez, A. \& Llorente-Cejudo, C. (2020). Marcos de Competencias Digitales para docentes universitarios: su evaluación a través del coeficiente competencia experta. Revista Electrónica Interuniversitaria de Formación del Profesorado, 23(3), 17-34.

\title{
Marcos de Competencias Digitales para docentes Universitarios: su evaluación a través del coeficiente competencia experta
}

Julio Cabero-Almenara, Julio Barroso-Osuna, Antonio Palacios-Rodríguez, Carmen Llorente-Cejudo

Universidad de Sevilla

\section{Resumen}

Las tecnologías se encuentran en el centro de una de las transformaciones más radicales que se han producido en la enseñanza universitaria, reclamando la atención del profesorado y de planes de formación del profesorado. Diferentes instituciones han propuesto una serie de marcos competenciales sobre las competencias prioritarias en las cuales deben formarse los docentes. El objetivo del trabajo es mediante juicio de expertos, elegir un marco de competencias que se convierta en el eje de una investigación más amplia: Diseño, producción y evaluación de t.MOOC para la adquisición por los docentes de "Competencias Digitales Docentes" (DIMOOC)". Se enviaron 369 correos a expertos, se recibieron 179 contestaciones aplicando el Coeficiente de Competencia Experta que hizo que nos el total fuese 148 expertos. Tras los resultados obtenidos, se puede constatar que ha sobresalido un marco de Competencia Digital Docente (CDD), el Marco de la Unión Europea de Competencia Digital Docente (DigCompEdu).

\section{Palabras clave}

Competencia digital; diseño de medios y materiales; índice de competencia experta.

\section{Contacto:}

Carmen Llorente-Cejudo, karen@us.es, Calle Pirotecnia s/n 41013, Sevilla, (España).

El presente artículo es parte del proyecto Diseño, producción y evaluación de t-MOOC para la adquisición por los docentes de competencias digitales docentes (RTI2018-097214-B-C31) financiado por el Ministerio de Ciencia, Innovación y Universidades. 


\title{
Digital Competency Frames for university teachers: evaluation through the expert competence coefficient
}

\begin{abstract}
The technologies are at the center of one of the most radical transformations that have occurred in university education, claiming the attention of teachers and teacher training plans. Different institutions have proposed a series of competency frameworks on the priority competences in which teachers should be trained. The objective of the work is through expert judgment, to choose a framework of competencies that becomes the focus of a broader investigation: Design, production and evaluation of t.MOOC for the acquisition by teachers of "Digital Teaching Competencies" (DIMOOC ) " 369 emails were sent to experts, 179 replies were received applying the Expert Competence Coefficient that made the total of 148 experts. After the results obtained, it can be seen that a framework of Digital Teaching Competency (CDD), the Framework of the European Union of Digital Teaching Competence (DigCompEdu), has excelled.
\end{abstract}

\section{Key words}

Digital competence; media and material design; expert proficiency index.

\section{Introducción}

Una de las transformaciones radicales que se ha producido en la enseñanza universitaria ha venido como consecuencia de la fuerte presencia que las Tecnologías de la Información y Comunicación están teniendo en dichas instituciones, como claramente se puede observar en el contexto español al analizar los sucesivos informes "Universitic" elaborados por el “Consejo de Rectores de las Universidades Españolas" (Gómez, 2016 \& 2017). Pero desgraciadamente, esta presencia no ha venido siempre acompañada, independientemente del nivel educativo, de planes de formación (Casal, Fernández-Morante \& Cebreiro, 2018; Suárez-Rodríguez, Almerich, Orellana \& Díaz-García, 2018; Silva et al., 2019); y como se ha señalado por algunos autores (Bernal-Bravo, Gozálvez, Gómez \& Masanet, 2019) desgraciadamente incluso muchas veces se les ha concedido poca importancia, incluso para aquellos que en un futuro se dedicarían a la docencia (Bernal-Bravo, Gozálvez, Hernando \& Masanet, 2019). Los motivos apuntados por los docentes de su baja formación, no se refieren al interés por las TIC, sino más como consecuencia de falta de apoyo institucional, tiempo, falta de recurso y la no existencia de planes de formación (Fernández-Márquez, LeivaOlivencia \& López-Meneses, 2017).

Por otra, parte cuando la formación se ha llevado a cabo, se ha realizado bajo modelos centrados más en aspectos instrumentales y tecnológicos, que en dimensiones pedagógicas y didácticas (Semerci \& Kemal, 2018; Cabero \& Martínez, 2019).

Este aspecto, hace que se vengan acuñando términos como el de Competencia Digital Docente (CDD), no refiriéndonos a procesos formativos únicamente centrados en el mero manejo instrumental de las tecnologías. La propia Comisión Europea (2016), señala que esta es una de las competencias claves que debe dominar la ciudadanía de forma general, y el profesorado de manera específica, en la sociedad del futuro. Tal es la significación que están adquiriendo la temática de las CDD, que se está convirtiendo en una línea consolidada de investigación en el contexto científico español (Rodríguez-García, Trujillo \& Sánchez, 2018), que van desde su descripción, a la búsqueda de instrumentos de diagnóstico (Esteve, Gisbert, 
\& Lázaro, 2016; Durán, Prendes \& Gutiérrez (2016a); INTEF, 2018; Tourón, Martín, Navarro, Pradas \& Íñigo, 2018; Padilla, Gámiz \& Romero, 2019; Salazar \& Gibrán, 2019), y su certificación (Durán, Prendes \& Gutiérrez, 2016b; Amaya, Salazar, Zúñiga, \& Ávila, 2018; Gudmundsdottir \& Hatlevic, 2018; Durán, Prendes \& Gutiérrez, 2019).

Ahora bien, ¿qué podemos entender por CDD. Y comenzando por la definición de “competencia digital" la Comisión Europea (2006) sugiere que es un uso seguro y crítico de las TIC de la Sociedad de la Información, "para el trabajo, el ocio y la comunicación. Se sustenta en las competencias básicas en materia de TCl: el uso de ordenadores para obtener, evaluar, almacenar, producir, presentar e intercambiar información, y comunicarse y participar en redes de colaboración" (15). Por otra parte, y abordando la definición de CDD y de acuerdo con Nieto, Pech \& Callejas (2017), se puede señalar que es una competencia transversal, que se relaciona con las otras grandes competencias docentes: saber, saber hacer, saber estar con otros y saber ser. A la hora de su definición, y asumiendo con Flores \& Roig (2019) que no es una tarea fácil, se puede concretar también de acuerdo con estos autores en que "es un tipo de competencia multidimensional y puede ser definida como la capacidad para movilizar aquellas habilidades y destrezas que permiten buscar, seleccionar críticamente, obtener y procesar información relevante haciendo uso de las tic para transformarla en conocimiento, al mismo tiempo que se es capaz de comunicar dicha información a través de la utilización de diferentes soportes tecnológicos y digitales, actuando con responsabilidad, respetando las normas socialmente establecidas y aprovechando estas herramientas para informarse, aprender, resolver problemas y comunicarse en distintos escenarios de interacción." (Flores \& Roig, 2019, 154).

En definitiva, como señala Durán $(2019,27)$ es un "conjunto de conocimientos, capacidades y actitudes necesarios para que un docente haga un uso efectivo de las TIC desde sus distintas vertientes (tecnológica, informacional, multimedia, comunicativa, colaborativa y ética), asumiendo criterios pedagógico-didácticos para una integración efectiva de las TIC en su experiencia docente y en general en cualquier situación educativa formal o no formal."

Para la adquisición de estas CDD, se han propuesto a nivel institucional desde diferentes instituciones una serie de marcos competenciales que sugieren las competencias prioritarias en las cuales deben formarse los docentes. Marcos que de acuerdo con los comentarios realizados por diferentes autores (Durán, Gutiérrez y Prendes, 2016; Marín, 2017; Cabero \& Martínez, 2019; Lázaro, Usart \& Gisbert, 2019; Loreli, Gámiz-Sánchez \& Romero-López, 2019; Rodríguez-García, Raso Sánchez y Ruiz-Palmero, 2019; Silva, Morales, Lázaro \& Gisbert, 2019; Cabero \& Palacios, 2020), podemos considerar como los más consolidados los siguientes:

- Marco de la Unión Europea de Competencia digital Docente.

- Marco de la "International Society for Technology in Education" (ISTE) para docentes.

- Marco de la Unesco competencial TIC para docentes.

- Marco común de competencia digital docente del "Instituto Nacional de Tecnología Educativa y de Formación del Profesorado" (INTEF).

- Marco de enseñanza digital del Reino Unido.

- Competencias TIC para el desarrollo profesional docente del Ministerio de Educación nacional de Colombia.

- Competencias y estándares TIC para la profesión docente del Ministerio de Educación de Chile. 
Marcos que persiguen reconocer la manera en que deben utilizarse e integrarse las Tecnologías en la enseñanza, identificar necesidades formativas y proponer itinerarios formativos personalizados (Flores-Lueg \& Roig Vila, 2016).

Marcos que presentan una serie de características específicas. Así, el "Marco Europeo de Competencia Digital del profesorado" (DigCompEdu), se articula alrededor de seis áreas competenciales diferenciadas (compromiso profesional, recursos digitales, pedagogía digital, evaluación y retroalimentación, empoderar a los estudiantes y facilitar la competencia digital a los estudiantes), llevando cada una serie de competencias que los "docentes deben poseer para fomentar estrategias de aprendizaje efectivas, inclusivas e innovadoras, utilizando herramientas digitales" (Redecker \& Punie, 2017, 4).

Al mismo tiempo el DigCompEdu propone seis niveles competenciales progresivos: novato, explorador, integrador, experto, líder y pionero; que sugieren diferentes niveles de manejo e integración educativa de las tecnologías en la práctica educativa.

Por otra parte, la Sociedad Internacional de Tecnología en Educación (ISTE) ha propuesto un marco competencial centrándose en las necesidades del estudiante del S. XXI, y de un docente caracterizado por ser activo e innovador en su actividad profesional de la enseñanza (Gutiérrez Castillo, Cabero Almenara, \& Estrada Vidal, 2017). En este caso se asumen siete roles o perfiles por los cuales puede pasar un docente en su desarrollo profesional y que suponen acciones diferentes a emprender por el docente, estos roles son: aprendices, líderes, ciudadanos, colaboradores, diseñadores, facilitadores y analistas. Pero dentro de los marcos uno que ha tenido bastante significación es el formulado por la UNESCO, en el cual se aboga por el conocimiento práctico de los beneficios que aportan las TIC a la educación. Insistiendo como los anteriores, en la necesidad de que sean dominados por los docentes para que ayuden a sus estudiantes a convertirse en ciudadanos colaborativos, creativos, innovadores, comprometidos y resolutivos (Rodríguez, Méndez, \& Martín, 2018). Su propuesta se articula alrededor de que los docentes adquieran diferentes competencias para: a) Comprender las TIC en la política educativa, b) Curriculum y evaluación, c) Pedagogía, d) Aplicación de habilidades digitales, e) Organización y administración, y f) Aprendizaje profesional.

En España, el Ministerio de Educación ha elaborado un "Marco Común de Competencia Digital Docente" cuyas últimas versiones se efectuaron en el año 2017 (INTEF, 2017a, 2017b). El proyecto se apoya en el elaborado por "DigComp" elaborado por la Unión Europea (Carretero, Vuorikari, \& Punie, 2017; Ferrari, 2013; Vuorikari, Punie, Carretero, \& Van-denBrande, 2016). La propuesta se articula alrededor de cinco áreas competenciales (a) Información y alfabetización informacional; b) Comunicación y colaboración; c) Creación de contenido digital; d) Seguridad; y e) Resolución de problemas) y veintiún competencias.

La propuesta identifica seis niveles competenciales, en una línea muy similar al DigCompEdu, identifica el nivel competencial digital docente, que comienzan en el nivel A1 y finalizan en el nivel máximo $\mathrm{C} 2$.

También el gobierno británico ha elaborado el suyo a través de la "Fundación para la Educación y la Enseñanza" (ETF) y la empresa JISC (Education and Training Foundation, 2019). El marco conforma siete áreas claves (a) Planificación Pedagógica; b) Enfoque Pedagógico; c) Empleabilidad del Alumnado; d) Enseñanza Específica; e) Evaluación; f) Accesibilidad e Inclusión; y g) Autodesarrollo. Para cada uno de ellos establece tres niveles: exploración, adaptación y líder.

En Latinoamérica se han elaborado dos grandes propuestas, una por el Ministerio de Educación colombiano y otra por el chileno, que en líneas generales persiguen fomentar el 
desarrollo profesional docente para la mejora de la innovación educativa con TIC (Fernanda et al., 2013). En el caso colombiano, el marco identifica cinco competencias que deben poseer los profesores para tener un uso eficaz de las TIC, y que concretan en: a) Tecnológica; b) Comunicativa; c) Pedagógica; d) Gestión; y e) Investigación. La propuesta también señala que en cada uno de ellos pueden darse tres niveles: explorador, integrador e innovador.

Finalmente el marco del Ministerio de Educación de Chile, presenta cinco dimensiones en una línea similar a la propuesta presentada en su momento de la Unesco: a) Técnica; b) Pedagógica; c) Gestión; d) Desarrollo y Responsabilidad; y e) Social, Ética y Legal (Elliot, Gorichon, Irigoin, \& Maurizi, 2011; Butcher, 2019).

\section{Metodología}

La investigación que se presenta se inserta en una más amplia denominada: "Diseño, producción y evaluación de t-MOOC para la adquisición por los docentes de "Competencias Digitales Docentes" (DIMOOC)" entre los objetivos que persigue se encuentra: "Crear y evaluar un entorno formativo bajo la arquitectura t-MOOC, para la capacitación del profesorado de niveles no universitarios en la adquisición de la competencia digital docente."

\section{Instrumento}

Y para su alcance lo primero que se ha llevado a cabo, para la elección del marco de competencias digitales es un juicio de expertos. Juicio de Expertos, que "consiste, básicamente, en solicitar a una serie de personas la demanda de un juicio hacia un objeto, un instrumento, un material de enseñanza, o su opinión respecto a un aspecto concreto" (Cabero \& Llorente, 2013, 14).

Estrategia que cada vez se está extendiendo más en la investigación-evaluación educativa (Robles \& Rojas, 2015; Galicia, Balderrama \& Edel, 2017) y está fuertemente asociado a los estudios Delphi (López Gómez, 2018). El problema con que nos encontramos es que el concepto de experto es bastante polisémico, y no existe una conceptualización unívoca del mismo que ayude a precisar sus características definitorias y lo que entendemos por ello. $Y$ como podemos imaginarnos los resultados que se obtengan dependerá directamente de la calidad de los expertos que se seleccionen para el proceso evaluativo, y para ello hay diferentes procedimientos que van desde contemplar el perfil del experto seleccionado a otros, más complejos como es el coeficiente de competencia experta (CCE) (Cabero \& Barroso, 2013; Cabero \& Infante, 2014; López Gómez, 2018).

En el presente estudio se establecieron dos mecanismos para su selección; en primer lugar, los seleccionamos teniendo en cuenta que cumplieran dos o más de los siguientes criterios:

- Impartir docencia en Universidades en las asignaturas de "Tecnología Educativa", "Nuevas Tecnologías aplicadas a la Educación”, o "Tecnologías de la Información y Comunicación Aplicadas a la Educación".

- Tener experiencia en el terreno de la formación del profesorado en TIC.

- Haber escrito algún artículo sobre alfabetización en tecnología educativa, competencias digitales, alfabetización audiovisual, en revistas españolas y latinoamericanas, en los últimos cinco años.

- Ser de distintas Universidades Españolas y Latinoamericanas.

- El haber colaborado anteriormente con nosotros en otros trabajos de investigación. que nos permitiera conocer su seriedad y profesionalidad. 
- Haber asistido a alguno de los tres últimos congresos de Edutec.

- Haber participado en algunos de los tres últimos congresos de CITEI, centrados en las tecnologías aplicadas a la educación infantil

Uno de los problemas asociados al juicio de experto se refiere al número de expertos que se necesitan para la aplicación, sin querer extendernos mucho en ello, indicar que las propuestas oscilan entre: 15 - 20 (Malla \& Zabala, 1978), 15 -35 (Landeta, 2002); o 15 - 25 (Witkin \& Altschuld, 1995). Pero como ya señalamos en otro momento, su número vendrá determinado por diferentes aspectos: disponer de diferentes expertos hacia la temática analizada, evitar el menor número de pérdida de sujetos si se consideran diferentes vueltas, el volumen de trabajo que seamos capaces de analizar, la facilidad con que podamos acceder a la información y la rapidez con que debamos ofrecer los resultados preliminares (Cabero \& Barroso, 2013). En nuestro caso, puesto que existían problemas para trabajar con una amplia base de datos, y solamente íbamos a hacer una vuelta, adoptamos la decisión de trabajar con el mayor número posible.

\section{Procedimiento}

El número de correos electrónicos que se mandaron, de acuerdo con los criterios inicialmente tenidos en cuenta, a expertos para que colaboraran en la investigación fue de 369 , de los cuales aproximadamente fueron devueltos, por no estar la dirección de correo correctamente planteada, o por haber cambiado de institución la persona. Finalmente, tras las dos semanas en las cuales mantuvimos el cuestionario abierto, recibimos 179 contestaciones.

Pero nuestro interés por afinar en el proceso de selección de los expertos finales fue aplicar el CCE (Cabero \& Barroso, 2013; Cabero \& Infante, 2014; López Gómez, 2018; Martínez \& otros, 2018), que se obtiene a partir de la autopercepción que el experto tiene sobre su nivel de conocimiento respecto a la temática analizada, así como de las fuentes que le permiten argumentar la decisión adoptada. Obteniéndose mediante las preguntas que se señalan en el Anexo.

Para su obtención se utiliza la fórmula: $K=1 / 2(K c+K a)$. Donde $K c$ es el "coeficiente de conocimiento", y se obtiene de la puntuación ofrecida directamente por el experto en la pregunta A del Anexo, y Ka, el coeficiente de argumentación, que se alcanzan sumando las opciones especificadas por el experto en la tabla de la pregunta B del Anexo.

Los valores que se utilizan para determinar la posición del experto son:

- $\quad 0,8<\mathrm{K}<1,0$ coeficiente de competencia alto

- $\quad 0,5<\mathrm{K}<0,8$ coeficiente de competencia medio

- $\mathrm{K}<0,5$ coeficiente de competencia bajo

La aplicación del valor 0.8 llevó a quedarnos con 148 expertos; es decir con el 82,68\% de los que cumplimentaron el cuestionario.

Indicar que el $84,5 \%(f=125)$ de los jueces tenían estudio de doctorado y el $15,5 \%(f=23)$ de master, que mayoritariamente trabajaban en un centro universitario $(f=140,94,6 \%)$, y que básicamente su actividad profesional era la enseñanza $(f=145,98 \%)$ seguida de las de gestión $(f=35,29,4 \%)$. 
Tabla 1.

Características de los jueces seleccionados a partir de CCE $\geq 0.8$.

\begin{tabular}{|c|c|c|c|}
\hline Dimensión & & $f$ & $\%$ \\
\hline \multirow{2}{*}{$\begin{array}{l}\text { ¿Ha impartido a lo largo de su vida profesional } \\
\text { asignatura/contenidos/materias/acciones formativas relacionadas } \\
\text { con el terreno de la Tecnología Educativa? }\end{array}$} & $\mathrm{Si}$ & 143 & 96,6 \\
\hline & No & 5 & 3,4 \\
\hline \multirow{2}{*}{$\begin{array}{l}\text { ¿Ha dirigido o participado en alguna investigación relacionada con } \\
\text { aspectos de la formación virtual, formación a distancia, formación } \\
\text { del profesorado en TIC, competencias digitales de los docentes y } \\
\text { estudiantes, alfabetización digital,,...? }\end{array}$} & $\mathrm{Si}$ & 145 & 98 \\
\hline & No & 3 & $2 \%$ \\
\hline \multirow{2}{*}{$\begin{array}{l}\text { ¿Ha realizado o participado en alguna publicación relacionada con } \\
\text { aspectos de la formación virtual, formación a distancia, formación } \\
\text { del profesorado en TIC, competencias digitales de los docentes y } \\
\text { estudiantes, alfabetización digital, ...? }\end{array}$} & $\mathrm{Si}$ & 145 & $98 \%$ \\
\hline & No & 3 & $2 \%$ \\
\hline \multirow{2}{*}{$\begin{array}{l}\text { ¿Ha impartido a lo largo de su vida profesional } \\
\text { asignatura/contenidos/materias/charlas/conferencias relacionadas } \\
\text { con la formación del profesorado en TIC, competencias digitales, o } \\
\text { alfabetización digital? }\end{array}$} & $\mathrm{Si}$ & 142 & 95,9 \\
\hline & No & 6 & 4,1 \\
\hline
\end{tabular}

Como se observa, en cierta medida la gran mayoría de los expertos identificados señalan que tenían experiencia de docencia, publicaciones e investigaciones en temática de las TIC y la competencia y alfabetización digital del profesorado.

El instrumento de recogida de información contenía dos grandes apartados, en el primero, se recogía información respecto algunas características del experto (titulación, actividad profesional, lugar en el que trabaja, ...) y se incorporaban también las preguntas destinadas a elaborar el CCE; y en el segundo, se le solicitaba que valorara los diferentes marcos de competencias Digitales Docentes formulados por diferentes instituciones:

- Marco de la Unión Europea de Competencia digital Docente (M1)

- Marco de la "International Society for Technology in Education" (ISTE) para docentes. (M2)

- Marco de la Unesco competencial TIC para docentes. (M3).

- Marco común de competencia digital docente del "Instituto Nacional de Tecnología Educativa y de Formación del Profesorado" (INTEF) (M4).

- Marco de enseñanza digital del Reino Unido (M5)

- Competencias TIC para el desarrollo profesional docente del Ministerio de Educación nacional de Colombia (M6).

- Competencias y estándares TIC para la profesión docente del Ministerio de Educación de Chile (M7).

Marcos que, de acuerdo con diferentes autores, podemos señalar como los más utilizados en el contexto internacional (Prendes \& Gutiérrez, 2013; Durán, Gutiérrez \& Prendes, 2016; Marín, 2017; Cabero \& Martínez, 2019; Lázaro, Usart\& Gisbert, 2019; Loreli, Gámiz-Sánchez \& 
Romero-López, 2019; Rodríguez-García, Raso Sánchez\& Ruiz-Palmero, 2019; Silva, Morales, Lázaro \& Gisbert, 2019; Cabero \& Palacios, 2020).

El instrumento se administró vía internet y puede observarse en la siguiente dirección web: https://docs.google.com/forms/d/e/1FAIpQLSecYR6TwXIqoZpTVk9qN49_ivPxOnaUIImX8jyZ tgnasRylYg/formResponse.

La aplicación se efectuó en el mes de enero de 2020.

\section{Resultados}

En primer lugar, presentaremos los valores alcanzados por los expertos en los coeficientes de conocimiento (Kc) y de argumentación (Ka), y se presentan los valores medios alcanzados; el primero se obtiene de la media en las puntuaciones indicadas por los expertos de 0-10, y los segundos, asignando la puntuación de 1 a la opción de respuesta "bajo", 2 a "medio" y 3 a "alto".

Tabla 2.

Medias alcanzadas en los coeficientes de conocimiento $(K c)$ y de argumentación (Ka).

\begin{tabular}{lcc}
\hline Variable & Media & D. típ \\
\hline Conocimiento & 8,26 & 1,38 \\
\hline Análisis teóricos realizado por usted & 2,54 & 0,59 \\
\hline Su experiencia obtenida de su actividad práctica & 2,76 & 0,52 \\
\hline Estudio de trabajo sobre el tema, de autores españoles. & 2,51 & 0,64 \\
\hline Estudio de trabajo sobre el tema, de autores extranjeros. & 2,47 & 0,64 \\
\hline Su propio conocimiento acerca del estado del problema en el extranjero & 2,20 & 0,70 \\
\hline Su intuición sobre el tema abordado. & 2,34 & 0,63 \\
\hline
\end{tabular}

Como se puede observar, las autovaloraciones que se asignaron los expertos podrían considerarse de altas y muy significativas; ello sugeriría que los criterios iniciales que utilizamos para su identificación podrían considerarse como válidos y significativos para el objetivo perseguido.

Presentado los datos que llevaron a seleccionar los expertos, se pasará a presentar el resultado de los valores asignados para los diferentes marcos por los expertos que superaron el valor de 0.8 en el CCE. Indicar que presentamos los valores medios alcanzados en el total y en cada una de los estándares del marco, aunque para su selección como nos interesa quedarnos con un marco, utilizaremos la puntuación media alcanzado para el mismo.

Tabla 3.

Medias y desviaciones de los diferentes marcos. 


\begin{tabular}{|c|c|c|}
\hline $\begin{array}{l}\text { MARCO EUROPEO DE COMPETENCIA DIGITAL DEL PROFESORADO } \\
\text { (UNIÓN EUROPEA) (M1) }\end{array}$ & M & D.típ. \\
\hline Compromiso profesional & 5,65 & 0,55 \\
\hline Recursos digitales & 5,65 & 0,63 \\
\hline Pedagogía digital & 5,68 & 0,59 \\
\hline Evaluación y retroalimentación & 5,52 & 0,63 \\
\hline Empoderar a los estudiantes & 5,58 & 0,64 \\
\hline Facilitar la competencia digital de los estudiantes & 5,61 & 0,57 \\
\hline TOTAL ESCALA & 5,62 & 0,41 \\
\hline \multicolumn{3}{|l|}{ ESTÁNDARES “ISTE” PARA DOCENTES M2) } \\
\hline Aprendices & 5,33 & 0,75 \\
\hline Líderes & 5,06 & 0,88 \\
\hline Ciudadanos & 5,36 & 0,85 \\
\hline Colaboradores & 5,27 & 0,81 \\
\hline Diseñadores & 5,21 & 0,95 \\
\hline Facilitadores & 5,41 & 0,87 \\
\hline Analistas & 5,10 & 0,92 \\
\hline TOTAL ESCALA & 5,25 & 0,65 \\
\hline \multicolumn{3}{|l|}{ MARCO COMPETENCIAL TIC PARA DOCENTES (UNESCO) (M3) } \\
\hline Comprender las TIC en la política educativa & 5,04 & 0,92 \\
\hline Curriculum y evaluación & 5,40 & 0,72 \\
\hline Pedagogía & 5,62 & 0,60 \\
\hline Aplicación de habilidades digitales & 5,52 & 0,71 \\
\hline Organización y administración & 5,13 & 0,94 \\
\hline Aprendizaje profesional & 5,51 & 0,67 \\
\hline TOTAL ESCALA & 5,37 & 0,56 \\
\hline \multicolumn{3}{|l|}{$\begin{array}{l}\text { MARCO COMÚN DE COMPETENCIA DIGITAL DOCENTE DEL INTEF } \\
(\text { ESPAÑA) }(M 4)\end{array}$} \\
\hline Información y alfabetización informacional & 5,44 & 0,74 \\
\hline Comunicación y colaboración & 5,56 & 0,66 \\
\hline Creación de contenidos digitales & 5,31 & 0,84 \\
\hline Seguridad & 5,29 & 0,92 \\
\hline Resolución de problemas & 5,43 & 0,73 \\
\hline TOTAL ESCALA & 5,41 & 0,59 \\
\hline
\end{tabular}




\begin{tabular}{lcc}
\hline Planificación pedagógica & 5,62 & 0,56 \\
\hline Enfoque pedagógico & 5,53 & 0,68 \\
\hline Empleabilidad del alumnado & 5,28 & 0,90 \\
\hline Enseñanza específica & 5,08 & 0,87 \\
\hline Evaluación & 5,36 & 0,72 \\
\hline Accesibilidad e inclusión & 5,54 & 0,74 \\
\hline Autodesarrollo & 5,43 & 0,76 \\
\hline TOTAL ESCALA & 5,40 & 0,55 \\
\hline $\begin{array}{l}\text { COMPETENCIAS TIC PARA EL DESARROLLO PROFESIONAL DOCENTE } \\
\text { (COLOMBIA) (M6) }\end{array}$ & \\
\hline Tecnológica & 5,31 & 0,71 \\
\hline Comunicativa. & 5,51 & 0,68 \\
\hline Pedagógica & 5,60 & 0,66 \\
\hline Gestión & 5,15 & 0,81 \\
\hline Investigación & 5,43 & 0,79 \\
\hline TOTAL ESCALA & 5,40 & 0,57 \\
\hline $\begin{array}{l}\text { COMPETENCIAS Y ESTÁNDARES TIC PARA LA PROFESIÓN DOCENTE } \\
\text { (CHILE) (M7) }\end{array}$ & \\
\hline Pedagógica & 5,48 & 0,76 \\
\hline Técnica o instrumental & 5,17 & 0,89 \\
\hline De gestión & 5,97 & 0,92 \\
\hline Social, ética y legal & 5,34 & 0,82 \\
\hline Desarrollo y responsabilidad social & 5,27 & 0.68 \\
\hline TOTAL ESCALA & & \\
\hline & & \\
\hline
\end{tabular}

Al ordenar de mayor a menor puntuación media alcanzada por cada marco la ordenación obtenida fue la siguiente:

5.56 Marco de la Unión Europea de Competencia digital Docente.

5.41 Marco común de competencia digital docente del "Instituto Nacional de Tecnología Educativa y de Formación del Profesorado".

5.40 Competencias TIC para el desarrollo profesional docente del Ministerio de Educación nacional de Colombia.

5.40 Marco de enseñanza digital del Reino Unido.

5.37 Marco de la Unesco competencial TIC para docentes.

5.27 Competencias y estándares TIC para la profesión docente del Ministerio de Educación de Chile. 
5.25 Marco de la "International Society for Technology in Education" (ISTE) para docentes.

Con el objeto de conocer si las valoraciones realizadas por los expertos también se quiso saber si había diferencias significativas entre las respuestas ofrecidas por los expertos que alcanzaron una puntuación menor, o mayor igual de 0.8 . Para ello la prueba estadística que utilizaremos será la U de Mann-Whitney (Siegel, 1976) así como la d de Cohen (1988) para analizar el tamaño de los efectos.

Se presentan las puntuaciones medias y desviaciones típicas alcanzadas por los expertos en función de tener una puntuación $\geq$ a 0.8 , o no.

Tabla 4.

Puntuaciones medias y desviaciones típicas obtenidas por los expertos en función del valor $\geq a$ 0.8 .

\begin{tabular}{lcccc}
\hline \multirow{5}{*}{} & \multicolumn{3}{l}{ Experto $\geq$ a 0.8} & \\
\cline { 2 - 5 } & Media & D. estándar & Media & D. estándar \\
\cline { 2 - 5 } M1 & 5,62 &, 41 & 5,31 &, 62 \\
\hline M2 & 5,25 &, 65 & 5,10 &, 87 \\
\hline M3 & 5,37 &, 56 & 5,37 &, 62 \\
\hline M4 & 5,41 &, 59 & 5,06 &, 74 \\
\hline M5 & 5,40 &, 55 & 5,21 &, 73 \\
\hline M6 & 5,40 &, 57 & 5,27 &, 81 \\
\hline M7 & 5,27 &, 68 & 5,29 &, 78 \\
\hline
\end{tabular}

Para analizar la Ho (hipótesis nula) referida a la no existencia de diferencias entre las puntuaciones asignadas por los expertos en función de haber obtenido, o no, a $\geq a$ a 0.8 , con un riesgo alfa de equivocarnos del 0.05 , aplicamos la $U$ de Mann-Whitney. Se presentan los rangos promedio y la suma de rangos, y los valores $U$ obtenidos para la aceptación o rechazo de la Ho.

Tabla 5.

Rangos promedio y suma de rango.

\begin{tabular}{cccc}
\hline Marco & Experto $\geq$ a 0.8 & Rango promedio & Suma de rangos \\
\hline M1 & Si & 95,87 & 14955,50 \\
\hline No & 69,65 & 1880,50 \\
\hline M2 & Si & 92,63 & 14449,50 \\
\hline
\end{tabular}




\begin{tabular}{cccc}
\hline Marco & Experto $\geq$ a 0.8 & Rango promedio & Suma de rangos \\
\hline & $\mathrm{No}$ & 88,39 & 2386,50 \\
\hline M3 & $\mathrm{Si}$ & 91,68 & 14302,00 \\
\hline & $\mathrm{No}$ & 93,85 & 2534,00 \\
\hline M4 & $\mathrm{Si}$ & 96,16 & 15001,00 \\
\hline $\mathrm{No}$ & $\mathrm{Ni}$ & 67,96 & 1835,00 \\
\hline & $\mathrm{No}$ & 94,02 & 14667,00 \\
\hline $\mathrm{M} 6$ & $\mathrm{Si}$ & 80,33 & 2169,00 \\
\hline & $\mathrm{No}$ & 92,26 & 14393,00 \\
\hline $\mathrm{M} 7$ & $\mathrm{Si}$ & 90,48 & 2443,00 \\
\hline & $\mathrm{No}$ & 91,16 & 14221,50 \\
\hline & & 96,83 & 2614,50 \\
\hline
\end{tabular}

Y, a continuación, lo que se refiere a los valores obtenidos en la U-Mann-Whitney.

Tabla 6.

Valores U de Mann Whitney.

\begin{tabular}{llllllll}
\hline & $M 1$ & $M 2$ & $M 3$ & $M 4$ & $M 5$ & $M 6$ & $M 7$ \\
\hline U de Mann-Whitney & 1502,5 & 2008,5 & 2056,0 & 1457,0 & 1791,0 & 2065,0 & 1975,5 \\
\hline W de Wilcoxon & 1880,5 & 2386,5 & 14302,0 & 1835,0 & 2169,0 & 2443,0 & 14221,5 \\
\hline$Z$ & $-2,427$ &,- 385 &,- 198 & $-2,582$ & $-1,248$ &,- 164 &,- 519 \\
\hline Sig asintótica &, 015 &, 700 &, 843 &, 010 &, 212 &, 870 &, 603 \\
\hline
\end{tabular}

Los valores obtenidos solo nos permiten rechazar la Ho formuladas a un nivel de significación del .05 o inferior, en el Marco 1 ("Marco de la Unión Europea de Competencia digital Docente") y M 4 ("Marco común de competencia digital docente del "Instituto Nacional de Tecnología Educativa y de Formación del Profesorado").

Por tanto, podemos concluir que las valoraciones realizadas por los diferentes expertos participantes en la investigación son diferentes en función de la puntuación de 0.08 alcanzada en el CCE.

Tales diferencias al aplicar el estadístico de Cohen, para analizar el tamaño del efecto, y de acuerdo con la propuesta realizada por el autor (Cohen, 1988), podemos considerarlas de altas (M1, M3, y M4) y moderadas (M2, M3, M4, M5 y M7).

Tabla 7.

Valores de Cohen. 
Estadístico de Cohen

\begin{tabular}{llll} 
M1 & 0.689 & M2 & \\
\hline M3 & M4 & 0.567 \\
\hline M5 & M6 & \\
\hline M7 & & \\
\hline
\end{tabular}

Realizado este análisis, se analiza si existen diferencias significativas entre las diferentes puntuaciones otorgadas por los jueces a los diferentes marcos. Y para ello se aplicaron dos estadísticos: en primer lugar, el de Friedman, que es una prueba no paramétrica alternativa al Análisis de la Varianza (Siegel, 1976; Gil, Rodríguez \& García, 1995), con el objeto de identificar qué marco había obtenido mayor rango entre los diferentes propuestos, y posteriormente analizaremos, mediante la prueba de rango con signo de Wilcoxon para muestras relacionadas (Siegel, 1976). Finalmente se aplicará la d de Cohen para conocer el tamaño de los efectos.

A continuación, se presentan los valores de rango promedio, para establecer el marco más considerado y la ordenación de todos, y si lo obtenido era significativo.

Tabla 8.

Estadístico de Friedman.

\begin{tabular}{llllll}
\hline \multicolumn{2}{l}{ Rango promedio } & & \multicolumn{3}{l}{ Prueba de Friedman } \\
\hline M1 & 5.05 & $M 2$ & 3.34 & $\mathrm{~N}$ & 148 \\
\hline M3 & 4.00 & $M 4$ & 3.92 & Chi-cuadrado & 67.562 \\
\hline M5 & 4.13 & $M 6$ & 4.04 & gl & 6 \\
\hline M7 & 3.51 & & & Sig. asintótica & ,000 \\
\hline
\end{tabular}

Lo obtenido indica, que al menos existen diferencias significativas $p \leq .000$, entre el primer rango obtenido (M1) y el último (M7); es decir, entre el mayor rango obtenido y el menor.

A continuación, pasaremos analizar, mediante la prueba de rango con signo de Wilcoxon entre el mayor y los restantes.

Tabla 9.

Prueba de rango con signo de Wilcoxon.

\begin{tabular}{lcl}
\hline Hipótesis nula $(\mathrm{Ho})$ contrastada & Sig & Decisión \\
\hline M1 con $\mathrm{M}_{2}$ & .000 & Rechazar Ho \\
\hline M1 con $\mathrm{M}_{3}$ & .000 & Rechazar Ho \\
\hline M1 con $\mathrm{M}_{4}$ & .000 & Rechazar Ho \\
\hline
\end{tabular}




\begin{tabular}{lll}
\hline M1 con M5 & .000 & Rechazar Ho \\
\hline M1 con M6 & .000 & Rechazar Ho \\
\hline M1 con M7 & .000 & Rechazar Ho \\
\hline
\end{tabular}

Los valores alcanzados nos llevan a rechazar las Ho, en consecuencia, apodemos señalar que hay diferencias significativas entre el rango mayor obtenido (M1) y los restantes a un nivel de significación de $\mathrm{p} \leq .05$.

Finalmente, para conocer el tamaño de tales diferencias aplicamos la d de Cohen, alcanzando los valores que se presentan a continuación.

Tabla 10.

Valores de Cohen.

Estadístico de Cohen

\begin{tabular}{llll}
\hline M1-M2 & 0.59 & $M 1-M_{3}$ & 0.39 \\
\hline M1-M4 & 0.39 & M1-M5 & 0.37 \\
\hline M1-M6 & 0.35 & M1-M7 & 0.51 \\
\hline
\end{tabular}

Valores que nos llevan a señalar que las diferencias encontradas suele ser bajas o moderadas.

\section{Discusión y conclusiones}

Las conclusiones del trabajo van en diferentes direcciones, y la primera de ellas se refiere a la significación del CCE como estrategia para la elección de los expertos. Pues como se ha puesto de manifiesto en el trabajo, las puntuaciones ofrecidas por los expertos, después de aplicarlo son diferentes. De todas formas, sería necesario contemplar que previamente debe haber un filtro previo, como el que se ha puesto en la presente investigación.

En el trabajo ha sobresalido un marco de CDD, el DigCompEdu, respecto a los demás, seguido del INETF; esto último, es lógico si se tiene en cuenta la inspiración que el segundo tiene del primero. Tal hallazgo, nos servirá en nuestro proyecto para el abordaje del plan de formación del profesorado desde la perspectiva seleccionada por los jueces, aunque también deben indicar a nuestras instituciones las directrices de por dónde deben establecer los planes de formación de los docentes en CDD.

De todas formas, es importante no confundir los resultados, con que los otros marcos no son significativos para adquirir las CDD, sino solo las preferencias otorgadas por los jueces; pues las similitudes entre los diferentes marcos son bastantes (Loreli, Gámiz-Sánchez \& RomeroLópez, 2019), y en nuestro estudio, aunque lo desconocemos, tales diferencias podrían deberse a la cumplimentación del instrumento por jueces españoles.

Lo comentado nos lleva sugerir diferentes líneas futuras de investigación, las cuales serían: replicar el estudio para a dos o tres vueltas donde progresivamente se vayan eliminando marcos de las posibles elecciones, ello exigiría una menor utilización de expertos y requeriría 
un compromiso previo de los mismos a la participación en la investigación en un tiempo mayor, y solicitar la nacionalidad, para su correlación con el marco elegido, y analizar si ello se convierte en un elemento distorsionante.

\section{Referencias}

Amaya, A., Salazar, M., Zúñiga, E. \& Ávila, A. (2018). Empoderar a los profesores en su quehacer académico a través de certificaciones internacionales en competencias digitales. Apertura, 10(1), 104-115. Doi: http://dx.doi.org/10.18381/Ap.v10n1.1174.

Bernal, C., González, V., Hernando A. \& Masanet, M.J. (2019). Relación entre formación universitaria y competencia mediática del profesorado. Revista Complutense de Educación, 30(4), 1113-1126. Doi: https://dx.doi.org/10.5209/rced.60188.

Butcher, N. (2019). Marco de competencias docentes en materia de TIC UNESCO. París: UNESCO.

Cabero, J. \& Infante, A. (2014). Empleo del método Delphi y su empleo en la investigación en comunicación y educación. Edutec. Revista Electrónica de Tecnología Educativa, 48. Recuperado de http://goo.gl/zDnTm9.

Cabero, J. \& Barroso, J. (2013). La utilización del juicio de experto para la evaluación de TIC: el Coeficiente de competencia experta. Bordón. Revista de Pedagogía, 65(2), 25-38.

Cabero, J. \& Llorente, M.C. (2013), La aplicación del juicio de experto como técnica de evaluación de las tecnologías de la información (TIC). Eduweb. Revista de Tecnología de Información y Comunicación en Educación, 7(2), 11-22.

Cabero, J. \& Martínez, A. (2019). Las tecnologías de la información y comunicación y la formación inicial de los docentes. Modelos y competencias digitales. Profesorado. Revista de Curriculum y Formación del Profesorado, 23(3), 247-268.

Cabero-Almenara, J., \& Palacios-Rodríguez, A. (2020). Marco Europeo de Competencia Digital Docente «DigCompEdu». Traducción y adaptación del cuestionario «DigCompEdu Check-In». EDMETIC, 9(1), 213-234. Doi: https://doi.org/10.21071/edmetic.vgi1.12462.

Carretero, S., Vuorikari, R., \& Punie, Y. (2017). DigComp 2.1: The Digital Competence Framework for Citizens With eight proficiency levels and examples of use. Luxembourg: Publication Office of the European Union. Doi: https://doi.org/10.2760/38842

Casal, L. Fernández-Morante, C. \& Cebreiro (2018). La competencia en TIC del profesorado no universitario. Revista Interuniversitaria de Investigación en Tecnología Educativa, 5, 2239. Doi: http://dx.doi.org/10.6018/riite/2018/334851.

Cohen, J. (1988). Statistical Power Analysis for the Behavioral Sciences (2nd ed.). Hillsdale, NJ: Lawrence Erlbaum Associates, Publishers.

Consejo de la Unión Europea. (2006). Recomendación del Parlamento Europeo y del Consejo de 18 de diciembre de 2006 sobre las competencias clave para el aprendizaje permanente. Bruselas: Diario Oficial de la Unión Europea.

Consejo de la Unión Europea. (2018). Recomendación del Consejo, de 22 de mayo de 2018, relativa a las competencias clave para el aprendizaje permanente. Bruselas: Diario Oficial de la Unión Europea.

Durán, M. (2019). Competencia Digital del Profesorado Universitario: Diseño y Validación de un Instrumento para la Certificación. Murcia. Universidad de Murcia-Escuela Internacional de Doctorado. Tesis doctoral inédita. 
Durán, M. C., Prendes, M.P.E. \& Gutiérrez, I. P. (2019). Certificación de la Competencia Digital Docente: propuesta para el profesorado universitario. RIED. Revista Iberoamericana de Educación a Distancia, 22(1), pp. 187-205. Doi: http://dx.doi.org/10.5944/ried.22.1.22069.

Durán, M., Gutiérrez, I., \& Prendes, M.P. (2016a). Análisis conceptual de modelos de competencia digital del profesorado universitario. Relatec. Revista Latinoamericana de Tecnología Educativa, 15(1), 97-114.

Durán, M., Gutiérrez, I., \& Prendes, M.P. (2016b). Certificación de la Competencia TIC del Profesorado Universitario. Diseño y validación de un instrumento. Revista Mexicana de Investigación Educativa, 21(6), 527-556.

Education and Training Foundation. (2019). Digital Teaching Professional Framework. Taking Learning to the Next Level. London: JISC.

Elliot, J., Gorichon, S., Irigoin, M., \& Maurizi, M. (2011). Competencias y Estándares TIC para la Profesión Docente. Santiago: Ministerio de Educación.

Esteve, F., Gisbert, M \& Lázaro, J.L. (2016). La competencia digital de los futuros docentes: ¿cómo se ven los actuales estudiantes de educación? Perspectivas Educacional. 55(2), 38-54.

Fernanda, M., Saavedra, C., De, M., Nacional, E., Del Pilar, P., Barrios, M. \& Zea, C. (2013). Competencias tic para el desarrollo profesional docente. Bogotá: Ministerio de Educación Nacional.

Fernández-Márquez, E., Leiva-Olivencia, J. \& López-Meneses, E. (2017). Competencias digitales en docentes de Educación Superior. Revista de Digital de Investigación en Docencia Universitaria, 12(1), 213-231.

Ferrari, A. (2013). DIGCOMP: A Framework for Developing and Understanding Digital Competence in Europe. Luxembourg: Publications Office of the European Union. Doi: https://doi.org/10.2788/52966.

Flores-Lueg, C., \& Roig Vila, R. (2016). Diseño y validación de una escala de autoevaluación de competencias digitales para estudiantes de pedagogía. Píxel-Bit, Revista de Medios y Educación, 12(48), 209-224. Doi: https://doi.org/10.12795/pixelbit.2016.i48.14

Galicia, L., Balderrama, J. \& Edel, R. (2017). Validez de contenido por juicio de expertos: propuesta de una herramienta virtual. Apertura, 9(2), 42-53. Doi: http://dx.doi.org/10.18381/Ap.v9n2.993.

Gudmundsdottir, G. B., \& Hatlevic, O. E. (2018). Newly qualified teachers' professional digital competence: implications for teacher education. European Journal of Teacher Education, 41(2), 214-231.

Gutiérrez Castillo, J. J., Cabero Almenara, J., \& Estrada Vidal, L. I. (2017). Diseño y validación de un instrumento de evaluación de la competencia digital del estudiante universitario.

INTEF (2017a). Marco Común de Competencia Digital Docente. Enero 2017. Madrid: Instituto Nacional de Tecnologías Educativas y Formación del Profesorado.

INTEF (2017b). Marco Común de Competencia Digital Docente. Octubre 2017. Madrid: Instituto Nacional de Tecnologías Educativas y Formación del Profesorado.

INTEF (2018). Portafolio de la competencia digital docente. Recuperado de http://enlinea.intef.es/courses/course-v1:SPOOC-INTEF+PortfolioCDD+2018_ED1/about Landeta, J. (2002). El método Delphi: una técnica de previsión del futuro. Barcelona: Ariel.

Lázaro, J. L., Usart, \& Gisbert, M. (2019). Assessing Teacher Digital Competence: the Construction of an Instrument for Measuring the Knowledge of Pre-Service Teachers. 
Journal of New Approaches in Educational Research, 8(1), 73-78. Doi: 10.7821/naer.2019.1.370.

López Gómez, E. (2018). El método Delphi en la investigación actual en educación: una revisión teórica y metodológica. Educación XX1, 21(1), 17-40. Doi: 10.5944/educXX1.15536.

Loreli, Gámiz-Sánchez \& Romero-López (2019). Niveles de desarrollo de la competencia digital docente: una mirada a marcos recientes del ámbito internacional. Innoeduca. International Journal Of Technology And Educational Innovation, 5(2), 140-150. Doi: 10.24310/innoeduca.2019.v5i2.5600.

Malla, F. \& Zabala, I. (1978). La previsión del futuro en la empresa (III): el método Delphi. Estudios Empresariales, 39, 13-24.

Marín, V. (2017). Diseño y evaluación de un instrumento de competencia digital docente. Facultad de Educación, Universidad de las Islas Baleares, tesis doctoral no publicada.

Nieto, E., Pech, S. \& Callejas, A. (2017). Evaluación de la competencia digital docente. TIC y enseñanza de idiomas. En Sumozas, R. \& Nieto, E. (coords.). Evaluación de la competencia digital docente, 17-33.

Robles, P. \& Rojas, M.D.C. (2015). La validación por juicio de expertos: dos investigaciones cualitativas en Lingüística aplicada. Revista Nebrija de Lingüística Aplicada, 18. Recuperado de https://www.nebrija.com/revistalinguistica/files/articulosPDF/articulo_55002aca89c37.pdf.

Rodríguez, M. D. M., Méndez, V. G., \& Martín, A. M. R. M. R. (2018). Alfabetización Informacional Y Competencia Digital En Estudiantes De Magisterio. Profesorado, Revista de Currículum y Formación Del Profesorado, 22(3), 253-270. Doi: https://doi.org/10.30827/PROFESORADO.V2213.8001

Rodríguez-García, A. M., Raso, F., \& Ruiz-Palmero, J. (2019). Competencia digital, educación superior y formación del profesorado : un estudio de meta-análisis en la web of science. Pixel-Bit, 54(4), 65-81. Doi: https://doi.org/10.12795/pixelbit.2019.i54.04

Semerci, A. \& Kemal, M. (2018). Examining High School Teachers' Attitudes towards ICT Use in Education. International Journal of Progressive Education, 14(2). Doi: 10.29329/ijpe.2018.139.7.

Siegel, S. (1976). Estadística no paramétrica aplicada a las ciencias de la conducta. México: Trillas.

Silva, J., Morales, M. J., Lázaro, J. L. \& Gisbert, M. (2019). La competencia digital docente en formación inicial: Estudio a partir de los casos de Chile y Uruguay. Archivos Analíticos de Políticas Educativas, 27(93). Doi: https://doi.org/10.14507/epaa.27.3822.

Suárez-Rodríguez, J., Almerich, G., Orellana, N., \& Díaz-García, I. (2018). A basic model of integration of ICT by teachers: competence and use. Educational Technology Research and Development, 66(5), 1165-1187. Doi: https://doi.org/10.1007/s11423-018-9591-0

Tourón, J., Martín, D., Navarro, E., Pradas, S., \& Íñigo, V. (2018). Validación de constructo de un instrumento para medir la competencia digital docente de los profesores (CDD). Revista Española de Pedagogía, 76(269), 25-54. Doi: https://doi.org/10.22550/REP76-12018-02.

Vuorikari, R., Punie, Y., Carretero, S., \& Van-den-Brande, G. (2016). DigComp 2.0: The Digital Competence Framework for Citizens. Luxembourg: Publication Office of the European Union. Doi: https://doi.org/10.2791/11517 
Witkin, B. R. \& Altschuld, J. W. (1995). Planning and conducting needs assessment: A practical guide. Thousand Oaks, Sage. 